\title{
WIND TUNNEL MEASUREMENTS AND CALCULATIONS OF AERODYNAMIC INTERACTIONS BETWEEN TILTROTOR AIRCRAFT
}

\author{
Wayne Johnson \\ Gloria K. Yamauchi \\ Army/NASA Rotorcraft Division \\ Moffett Field, California
}

\author{
Michael R. Derby \\ Alan J. Wadcock \\ Aerospace Computing, Inc. \\ Moffett Field, California
}

\begin{abstract}
Wind tunnel measurements and calculations of the aerodynamic interactions between two tiltrotor aircraft in helicopter mode are presented. The measured results include the roll moment and thrust change on the downwind aircraft, as a function of the upwind aircraft position (longitudinal, lateral, and vertical). Magnitudes and locations of the largest interactions are identified. The calculated interactions generally match the measurements, with discrepancies attributed to the unsteadiness of the wake and aerodynamic forces on the airframe. To interpret the interactions in terms of control and power changes on the aircraft, additional calculations are presented for trimmed aircraft with gimballed rotors.
\end{abstract}

\begin{tabular}{llll} 
& \multicolumn{1}{c}{ Notation. } & $\mathrm{T}$ & aircraft thrust \\
$\mathrm{A}$ & rotor disk area, $\pi \mathrm{R}^{2}$ & $\mathrm{~V}$ & wind tunnel speed \\
$\mathrm{c}_{\mathrm{ref}}$ & blade reference chord & $\mathrm{x}$ & longitudinal distance between aircraft \\
$\mathrm{C}_{\mathrm{Mx}}$ & aircraft roll moment coefficient, & y & lateral distance between aircraft \\
& $\mathrm{M}_{\mathrm{X}} / \rho \mathrm{A}(\Omega \mathrm{R})^{2} \mathrm{R}$ & $\mathrm{z}$ & vertical distance between aircraft \\
$\mathrm{C}_{\mathrm{P}}$ & aircraft power coefficient, $\mathrm{P} / \rho(\Omega \mathrm{R})^{3} \mathrm{~A}$ & $\mu$ & advance ratio, $\mathrm{V} / \Omega \mathrm{R}$ \\
$\mathrm{C}_{\mathrm{T}}$ & aircraft thrust coefficient, $\mathrm{T} / 2 \rho(\Omega \mathrm{R})^{2} \mathrm{~A}$ & $\rho$ & air density \\
$\mathrm{D}$ & rotor diameter, $2 \mathrm{R}$ & $\sigma$ & rotor solidity, $\mathrm{Nc} \mathrm{c}_{\text {ref }} / \pi \mathrm{R}$ \\
$\mathrm{M}_{\mathrm{X}}$ & aircraft roll moment & $\Omega$ & rotor rotational speed
\end{tabular}

$\mathrm{N} \quad$ number of blades

P aircraft power

$\mathrm{r} \quad$ blade radial station ( 0 to $\mathrm{R})$

$r_{\mathrm{c}} \quad$ vortex core radius

$\mathrm{R} \quad$ blade radius

s wing semispan

-Presented at the 41st Aerospace Sciences Meeting and Exhibit, Reno, Nevada, January 6-9, 2003. Copyright (C) 2003 by the American Institute of Aeronautics and Astronautics, Inc. No copyright is asserted in the United States under Title 17, U.S. Code. The U.S. Government has a royalty-free license to exercise all rights under the copyright claimed herein for Governmental purposes. All other rights are reserved by the copyright owner.

\section{Introduction}

The tiltrotor aircraft configuration has the potential to revolutionize air transportation by providing an economical combination of vertical take-off and landing capability with efficient, high-speed cruise flight. In order to achieve the goal of a major impact on transportation systems, it will be necessary to understand, and to be able to predict, the aerodynamic interactions involving tiltrotors. NASA Ames Research Center is conducting a series of wind tunnel tests investigating the aerodynamic interactions of tiltrotors, including interactions between multiple aircraft, the ground, and structures. This paper presents wind tunnel measurements and calculations of the aerodynamic interactions between two tiltrotor aircraft operating in helicopter mode. 
A series of aerodynamic interaction tests are being conducted using model tiltrotors in the Army 7- by 10Foot Wind Tunnel at NASA Ames Research Center. Tests completed to date include:

a) Terminal area operations: aerodynamic interactions between two tiltrotor aircraft, primarily at 47 knots.

b) Terminal area operations: two aircraft, with low thrust on the downwind aircraft; with and without ground plane; flow visualization includes oil flow and tufts on ground plane.

c) Hover and low speed: one aircraft, in and out of ground effect; including measurements of the outflow velocity.

d) Single rotor (a helicopter rotor or one tiltrotor) upstream of a tiltrotor aircraft that is at low thrust; at 10 knots, with and without ground plane.

e) City operations: one aircraft in vicinity of a building; at 20 knots, including smoke flow visualization.

f) Baseline aerodynamics: one aircraft; constant thrust velocity sweeps up to 70 knots, and hover collective sweeps; PIV measurements of wake flow field.

g) Large yaw angles: in preparation for future test of airframe/rotor interference using a 0.25 -scale model, assessment of mounting strut interference effects.

For the above tests the tiltrotor model was in helicopter configuration, and aircraft forces and moments were measured in all cases. The speeds quoted above are equivalent full scale values. This paper presents results from just the first of the above subjects: the aerodynamic interactions between two tiltrotor aircraft. Such interaction may be expected to be an important factor in effective terminal area operations of tiltrotors.

Calculations of the aerodynamic interactions were performed using CAMRAD II, which is a modern rotorcraft comprehensive analysis that has advanced models intended for application to tiltrotor aircraft as well as helicopters. The objectives of the calculations were to first establish how well the wind tunnel measurements can be predicted using current wake models; and then to interpret the interactions in terms of control and power changes on the aircraft.

\section{Test and Model Description}

Two tiltrotor models were installed in the Army 7by 10-Foot Wind Tunnel at NASA Ames Research Center, as shown in figure 1. The key geometric parameters were chosen to be representative of tiltrotor designs: rotor planform and twist, the rotor/rotor separation, and the rotor/wing separation. The diameter of the rotors was $0.7812 \mathrm{ft}$, and the wing span was $0.9706 \mathrm{ft}$. This corresponds to a scale of about $1 / 32$ for the XV-15 or BA609; $1 / 49$ for the V-22; and 1/110 for a 100-passenger tiltrotor design.

The three-bladed rotors had counter-clockwise rotation on the right rotor, clockwise rotation on the left rotor. The tapered blades had a thrust-weighted solidity of $\sigma=0.102$ (with $25.5 \%$ root cutout); a total twist of $-47.5 \mathrm{deg}$ ( $-8 \mathrm{deg}$ from $75 \%$ radius to the tip); and airfoil thickness ratios of $28,18,12$, and $9 \%$ at $\mathrm{r} / \mathrm{R}$ $=0.25,0.50,0.75$, and 1.00 respectively. The wing was machined aluminum, with zero flap deflection. The wing semispan was $0.4853 \mathrm{ft}$. The hub and control system were commercially available radio-control model helicopter tail rotor assemblies. The rotors had collective pitch control, allowing trim of aircraft thrust and roll moment. The rotors did not have flap or lag hinges, or a gimbal, and did not have cyclic pitch control. Hence the rotors operated with some hub moment in edgewise flight (helicopter mode forward flight). The blade weight gives a Lock number of about 7.6. Figure 2 is a drawing of the tiltrotor model. A fuselage (including tail) was available, but not used for the investigation reported here. Each aircraft had a 6component balance to measure total aircraft forces and moments. The sting mount attached to a taper socket (shown aft of the wing in figure 2), which was attached to the balance. The electric motor (shown forward of the wing in figure 2) was on the metric side of the balance. The design rotational speed of the rotors was $6355 \mathrm{rpm}$, corresponding to a tip speed of $263 \mathrm{ft} / \mathrm{sec}$. The Reynolds number based on the blade tip chord and speed was about 63000 .

The tiltrotor aerodynamic interaction investigation was conducted with two tiltrotor models, the downwind aircraft operating in the wake of the upwind aircraft. The aircraft were in helicopter configuration (nacelle angle $90 \mathrm{deg}$ ), with the rotor shafts vertical and the wing at zero angle of attack and yaw. The downwind aircraft was mounted on a fixed sting. The upwind aircraft was mounted on a traverse mechanism (figure 1), allowing variation of its position in all three directions: longitudinal, lateral, and vertical $(\mathrm{x}, \mathrm{y}, \mathrm{z})$ relative to the downwind aircraft. With the upwind aircraft in the extreme top/port position and its rotors not turning, the downwind aircraft was trimmed to a specified thrust and to approximately zero roll moment (for each longitudinal position tested). The collective pitch angles of the rotors on the downwind aircraft were then kept fixed as the position of the upwind aircraft was varied in the lateral-vertical direction. The control of the upwind aircraft was always adjusted as required to maintain a 
specified thrust and zero roll moment. Thus the roll moment and thrust of the downwind aircraft are the primary measures of the interaction.

The upwind aircraft position $(\mathrm{x}, \mathrm{y}, \mathrm{z})$ was measured relative to the center of the downwind aircraft. The longitudinal separation $\mathrm{x}$ is specified in terms of the rotor diameter $\mathrm{D}$, measured between corresponding points on each aircraft. The $\mathrm{y}$ and $\mathrm{z}$ positions are specified in terms of the aircraft semispan s. The lateral position $\mathrm{y}$ is positive with the upwind aircraft to starboard, and the vertical position is positive with the upwind aircraft above the downwind aircraft. The coefficients used are defined as follows:

$$
\begin{aligned}
& \mathrm{C}_{\mathrm{T}} / \sigma=\mathrm{T} / 2 \rho \mathrm{A}(\Omega \mathrm{R})^{2} \sigma \\
& \mathrm{C}_{\mathrm{Mx}} / \sigma=\mathrm{M}_{\mathrm{X}} / \rho \mathrm{A}(\Omega \mathrm{R})^{2} \mathrm{R} \sigma
\end{aligned}
$$

where $\mathrm{T}$ and $\mathrm{M}_{\mathrm{x}}$ are the total aircraft thrust and moment. Note that the thrust coefficient is based on the area of both rotors, 2A. The roll moment is positive for roll right. The balance measured the load on the entire aircraft, including the wing. The wing download for the operating conditions considered is estimated to be less than $10 \%$ of the rotor thrust.

\section{Wind Tunnel Test Results}

The test results reported here consist of four runs (described in table 1), at nominal longitudinal separation distances of $\mathrm{x} / \mathrm{D}=2.5,5.0$, and 10.0 (two runs at $\mathrm{x} / \mathrm{D}=$ 2.5). The wind tunnel speed gave an advance ratio of $\mu=0.10$, corresponding to about 47 knots full scale. The rotor thrust was about $\mathrm{C}_{\mathrm{T}} / \sigma=0.12$, which was below stall for these small rotors (based on power characteristics).

\begin{tabular}{|c|c|c|c|c|}
\hline run number & 118 & 122 & 123 & 126 \\
\hline points & 77 & 87 & 87 & 87 \\
\hline nominal $\mathrm{x} / \mathrm{D}$ & 2.5 & 2.5 & 5.0 & 10.0 \\
\hline actual x/D & 2.54 & 2.54 & 5.08 & 10.16 \\
\hline$\mu$ & 0.100 & 0.100 & 0.100 & 0.100 \\
\hline \multicolumn{5}{|c|}{ downwind aircraft } \\
\hline $\mathrm{rpm}$ & 6356 & 6367 & 6258 & 6365 \\
\hline \multicolumn{5}{|c|}{ reference condition } \\
\hline $\mathrm{C}_{\mathrm{T}} / \sigma$ & 0.121 & 0.122 & 0.120 & 0.122 \\
\hline $\mathrm{C}_{\mathrm{Mx}} / \sigma$ & 0.0085 & -0.0043 & -0.0043 & -0.0056 \\
\hline \multicolumn{5}{|c|}{ upwind aircraft } \\
\hline $\begin{array}{l}\text { rpm } \\
\text { trim conditior }\end{array}$ & 6319 & 6353 & 6337 & 6349 \\
\hline $\mathrm{C}_{\mathrm{T}} / \sigma$ & 0.121 & 0.121 & 0.121 & 0.121 \\
\hline
\end{tabular}

Table 1. Test conditions.
Three data points were taken with the upwind aircraft at the reference position (extreme top/port, its rotors not turning). Then 87 points (except for run 118) were taken with the upwind aircraft traversing the grid shown in figure 3 . The grid was scaled with the wing semispan $\mathrm{s}$. The lateral position extended from $\mathrm{y} / \mathrm{s}=-7$ (forward aircraft to port) to $\mathrm{y} / \mathrm{s}=+3$, the vertical position from $\mathrm{z} / \mathrm{s}=-1$ (forward aircraft below) to $\mathrm{z} / \mathrm{s}=+4$. The resolution was 1 semispan, except additional points giving a vertical resolution of $1 / 2$ semispan for $\mathrm{y} / \mathrm{s}=$ \pm 2 . During the traverse over this grid, the mean operating condition values (rotor speed, advance ratio, and upwind aircraft thrust) exhibited an rms variation of about $1 \%$. Note in table 1 that the downwind aircraft in the reference condition does not quite have zero roll moment. Hence the roll moment data presented are relative to the roll moment at this reference condition. Figure 4 illustrates the relative location of the two aircraft for lateral positions of $\mathrm{y} / \mathrm{s}=0,-2$, and -4 .

Figure 5 shows the measured roll moment on the downwind aircraft, for $\mathrm{x} / \mathrm{D}=2.5,5.0$, and 10.0. The largest interaction occured for lateral separation of $\mathrm{y} / \mathrm{s}=$ \pm 2 , and $\mathrm{z} / \mathrm{s}$ about 1 (upwind aircraft above downwind aircraft). For $y / s=-2$, the left rotor of the downwind aircraft was aligned with the right rotor of the upwind aircraft (figure 4b). Hence the downwind left rotor was in the downwash of the upwind aircraft, which produced a reduction in thrust on the left rotor, hence a roll moment to the left (negative). There was also a local maximum of the interaction at $\mathrm{y} / \mathrm{s}=-4.5$. At $\mathrm{y} / \mathrm{s}=$ -4.5 , the left rotor of the downwind aircraft was outboard of the upwind aircraft, so the downwind left rotor was in an upwash, producing a thrust increase and a roll moment to the right (positive).

Figure 6 shows the measured change of the thrust on the downwind aircraft, for $\mathrm{x} / \mathrm{D}=2.5,5.0$, and 10.0. The largest interaction occured for $\mathrm{y} / \mathrm{s}=0$, when the downwind aircraft was directly behind the upwind aircraft. The average roll moment in this condition was zero, but there was a significant thrust reduction, because the downwind aircraft was operating in the downwash field of the upwind aircraft. At $\mathrm{y} / \mathrm{s}=-4.5$ there was a thrust increase, because of the upwash from the upwind aircraft. Note that the trim thrust value was $\mathrm{C}_{\mathrm{T}} / \sigma=0.12$, so the variations shown in figure 6 are significant.

Figures 5 and 6 both show a generally symmetric variation with lateral separation $y$. Such symmetry is expected, so it serves to confirm the quality of the data. As the longitudinal separation increased, the interaction became weaker, but was still relatively strong at $\mathrm{x} / \mathrm{D}=$ 10.0. The interaction moved to slightly larger $\mathrm{y} / \mathrm{s}$, indicating a lateral spreading of the wake; and to larger 
$\mathrm{z} / \mathrm{s}$, indicating downward convection of the wake (so the upwind aircraft was higher at the maximum interaction). Similar wake behavior was observed in lidar (light detecting and ranging) wake measurements of the XV-15 (reference 1). In helicopter mode at 60 and 90 knots, the wake was found to consist of a pair of vortices separated by the distance between the advancing blade tips. For these speeds, the decay and separation seemed to depend on time not distance. At 90 knots the wake maintained strength to $x / D=40$ or so, with little spreading. At 60 knots and $x / D=10$, the lateral separation was roughly 1.25 span, and the wake was just starting to decay.

\section{$\underline{\text { Rotorcraft Analysis }}$}

The aerodynamic interaction was calculated using the rotorcraft comprehensive analysis CAMRAD II, which is an aeromechanical analysis of helicopters and rotorcraft that incorporates a combination of advanced technologies, including multibody dynamics, nonlinear finite elements, and rotorcraft aerodynamics. CAMRAD II is described in references 2-4. The wake model for tiltrotor performance and airloads calculations has been the subject of recent correlation work, summarized in reference 5 .

For the present investigation, four rotors were modelled, with fully interacting wakes. The blades were assumed to be rigid (no gimbal and no elastic deflection), with no cyclic pitch and zero shaft angle. The aerodynamic model used lifting-line theory with a vortex wake calculation of the induced velocity. The blade aerodynamic surfaces were represented by 10 panels, from the root cutout of $r / R=0.255$ to the tip, with panel widths varying from $0.101 \mathrm{R}$ inboard to 0.040R at the tip. The drag coefficients in the airfoil tables were corrected to the lower Reynolds number of the model, using a factor equal to the Reynolds number ratio to the 1/5-power. Inboard stall delay was accounted for, as described in reference 5. A full free-wake analysis was performed, calculating the distorted geometry of tip vortices from each of the three blades on all four rotors. Calculations are only presented for $\mathrm{x} / \mathrm{D}=2.5$, with 16 revolutions of wake retained behind each rotor.

The wake model used was developed for the prediction of helicopter and tiltrotor performance, airloads, and structural loads. For such tasks many details of the wake model can have a significant influence on the calculated results. In contrast, because the aerodynamic phenomenon of interest here is the influence of a chaotic wake well downstream of the rotors generating the wake, most wake parameters had little effect on the downstream aircraft loads. A parameter that was found to have an influence was the rate of growth of the tip vortex viscous core. The calculations assumed a linear growth of core radius $r_{c}$ with wake age $\phi$ :

$$
\mathrm{r}_{\mathrm{c}} / \mathrm{c}=\mathrm{r}_{\mathrm{c} 0} / \mathrm{c}+\left(\phi / \phi_{1}\right)
$$

where $\mathrm{c}$ is the blade chord, and $\phi_{1}$ is the wake age where the core increment equaled the chord. An initial value of $\mathrm{r}_{\mathrm{c} 0} / \mathrm{c}=1.0$ was used, with a maximum core radius of $\mathrm{r}_{\mathrm{c}} / \mathrm{c}=10$. The baseline calculations used $\phi_{1}=1.5$ revolutions.

The calculations were performed in the same manner as the test procedures. The collective pitch of the downwind rotors was fixed at a value that gave the measured reference thrust and zero roll moment without the interaction of the upwind rotors. During the traverse, the upwind rotors were trimmed to the measured thrust $\left(\mathrm{C}_{\mathrm{T}} / \sigma=0.118\right)$ and zero roll moment. The calculations were performed for a lateral and vertical resolution of $1 / 2$ semispan. The calculated thrust presented is the sum of the thrust of the two downwind rotors. The calculated roll moment presented is that produced by the thrust of the two downwind rotors, plus the roll moments at the hubs of these two rotors. The analysis did not include a wing or fuselage.

\section{Correlation Results}

Figure 7 compares the measured and calculated roll moment on the downwind aircraft, for $\mathrm{x} / \mathrm{D}=2.5$ and $\mu=0.1$. The measured results are the average of the data for positive and negative $y$ (with appropriate sign changes), from run 122. The calculations exhibit the maxima at about $\mathrm{y} / \mathrm{s}=2$ and 4.5 , as observed in the test. The magnitude of the peak moment at $\mathrm{y} / \mathrm{s}=2$ matches well, although the calculated shape of the interaction region is different. The magnitude of the peak moment at $\mathrm{y} / \mathrm{s}=4.5$ is underpredicted. The calculations also show a local maximum at $\mathrm{y} / \mathrm{s}=1$ and $\mathrm{z} / \mathrm{s}=2.5$, which is not found in the measurements. It is believed that this discrepancy is associated with the unsteadiness of the flow field. The calculations were performed assuming that the wake geometry and resulting loading were perfectly periodic, but for the downwind aircraft directly behind the upwind aircraft (y near zero) a converged periodic solution became increasingly difficult to obtain.

Figure 8 compares the measured and calculated thrust change on the downwind aircraft, for $\mathrm{x} / \mathrm{D}=2.5$ and $\mu=$ 0.1 . The measured and calculated results show similar patterns, but the magnitude of the calculated thrust change is about one-half that measured. This difference perhaps reflects the influence of the wake on the wing and body of the aircraft, which were not included in the analysis.

American Institute of Aeronautics and Astronautics 
Figure 9 shows top and side views of the calculated wake geometry, for $\mathrm{y} / \mathrm{s}=2$ and $\mathrm{z} / \mathrm{s}=1$. For clarity, only one of the three tip vortices from each rotor is plotted. Note that the outer two rotors show relatively little downward convection of the wake. The wake from the port upwind rotor is ingested into the starboard downwind rotor, and the wakes from both rotors are convected downward faster. Preliminary examination of PIV measurements of the wake formation at 2.5D downstream of a single tiltrotor model (without the downwind aircraft) showed two super-vortices, with a lateral separation of about 1.20 times the aircraft span (between rotor edges). The calculated wake geometry showed a similar magnitude of lateral spreading of the wake.

Figure 10 examines the influence of the tip vortex core growth rate on the calculated roll moment, for $y / s$ $= \pm 2$. The baseline calculations used $\phi_{1}=1.5$ revs; the faster and slower growth cases were for $\phi_{1}=1.0$ and 2.0 respectively. Faster rate of core growth had little influence. With a slower rate of core growth, hence stronger interactions of the tip vortices in the wake, the calculated results are more erratic, reflecting a more chaotic wake geometry. The trend and magnitude of the calculated roll moment was about the same even with the slower core growth, but the sensitivity suggests that the unsteadiness of the interaction be further investigated.

Figure 11 shows the influence of the rotor hub moment on the aircraft roll moment, for $y / s= \pm 2$. The baseline results are for the rigid rotor, hence include the hub moments. The roll moment obtained considering just the rotor thrust changes is also shown, and indicates that the hub moment contribution to the total is small. Figure 11 also shows the aircraft roll moment calculated with a gimballed rotor, for which the hub moment is zero. The introduction of a gimbal has some influence, because the flapping of the rotor relative to the shaft is not zero (cyclic pitch was still not used). The influence of the Reynolds number correction was also examined, and was found to be small, since these interactions involve principally lift changes on rotors operating below stall.

In order to interpret the magnitude of the interactions observed, calculations were performed of the control required to maintain trim of the downwind aircraft. For these calculations a gimballed rotor was used, and the Reynolds number correction omitted. The geometry of the rotor and aircraft were not changed. Both aircraft were trimmed to $\mathrm{C}_{\mathrm{T}} / \sigma=0.120$ and zero roll moment. Figures $12 \mathrm{a}$ and $12 \mathrm{~b}$ show the calculated lateral control (differential collective) and collective control changes on the downwind aircraft, for $\mu=0.1$ and $\mathrm{x} / \mathrm{D}=2.5$, when the downwind aircraft is trimmed to constant thrust and zero roll moment. The lateral control (differential collective) change reflects the roll moment of the untrimmed aircraft, and ranges from -1.9 to $0.35 \mathrm{deg}$. The collective change reflects the aircraft thrust, and ranges from -0.8 to $2.9 \mathrm{deg}$. These control increments produced by the interaction are significant, but within the control authority of typical tiltrotor designs. Figure $12 \mathrm{c}$ shows the influence of the interaction on performance. The calculated change of the power coefficient ranges from $\Delta \mathrm{C}_{\mathrm{P}} / \sigma=-0.0020$ to 0.0065 , relative to a baseline $\mathrm{C}_{\mathrm{P}} / \sigma=0.0180$. (For a $40000 \mathrm{lb}$ tiltrotor, the aircraft power change is -600 to $1800 \mathrm{hp}$.) The power change is also a reflection of the thrust change of the untrimmed aircraft. Such performance changes are interesting, but probably of limited practical utility.

\section{Concluding Remarks}

Wind tunnel measurements and calculations of the aerodynamic interactions between two tiltrotor aircraft in helicopter mode have been presented. The measured roll moment on the downwind aircraft was largest when the left rotor of the downwind aircraft was operating in the downwash from the right rotor of the upwind aircraft (or the downwind right rotor operating in the downwash from the upwind left rotor). The measured thrust decrease was largest when the downwind aircraft was directly behind the upwind aircraft. There was also a local maximum in the roll moment, and a thrust increase, when the downwind aircraft was outboard of the upwind aircraft.

The calculated roll moment on the downwind aircraft matched the magnitude and location of the measured peak. The calculations also showed a local maximum in roll moment, not observed in the test, when the downwind aircraft was nearly aligned with the upwind aircraft. This discrepancy may be associated with the unsteady nature of the wake. The calculated thrust change on the downwind aircraft matched the pattern of the measurements, but the magnitude was smaller than measured, perhaps because the forces on the wing and body of the aircraft were not included in the analysis.

Calculations were performed of the control required to maintain trim of the downwind aircraft, including gimballed rotors. The lateral control (differential collective) reflected the roll moment on the untrimmed aircraft, and the collective control and power changes reflected the thrust change on the untrimmed aircraft. The control increments produced by the interaction were significant, but within the control authority of typical tiltrotor designs. 


\section{$\underline{\text { References }}$}

1) Schillings, J.J.; Ferguson, S.W.; Brand, A.G.; Mullins, B.R.; Libby, J.; "Wake Vortex Measurements of the XV-15 Tiltrotor Using a Mobile Ground-Based Lidar System." AHS International 57th Annual Forum Proceedings, Washington, D.C., May 2001.

2) Johnson, W. "Technology Drivers in the Development of CAMRAD II." American Helicopter Society Aeromechanics Specialists Conference, San Francisco, California, January 1994.

3) Johnson, W. "A General Free Wake Geometry Calculation for Wings and Rotors." American Helicopter Society 51st Annual Forum Proceedings, Fort Worth, Texas, May 1995.

4) Johnson, W. "Rotorcraft Aerodynamics Models for a Comprehensive Analysis." AHS International 54th Annual Forum Proceedings, Washington, D.C., May 1998.

5) Johnson, W. "Influence of Wake Models on Calculated Tiltrotor Aerodynamics." American Helicopter Society Aerodynamics, Acoustics, and Test and Evaluation Technical Specialists Meeting, San Francisco, CA, January 2002.

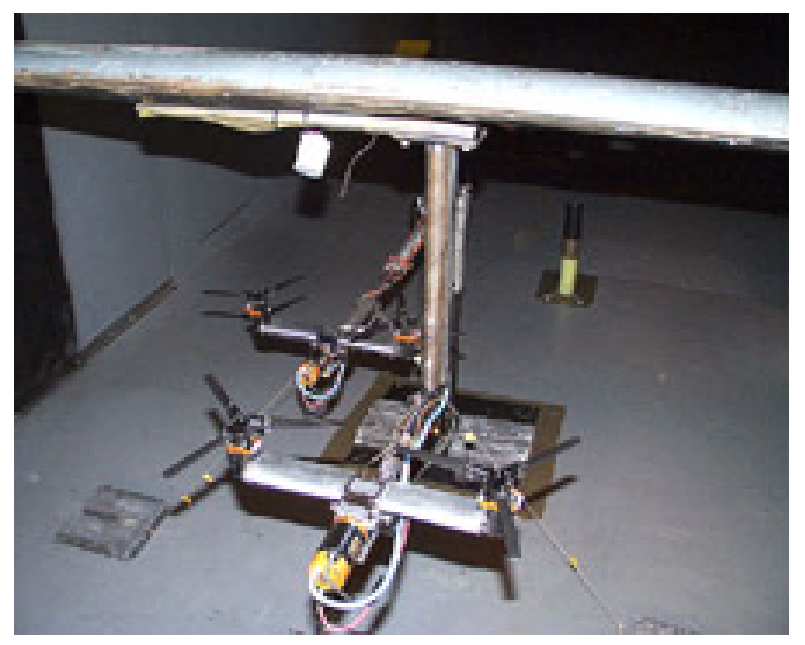

Figure 1. Tiltrotor models in the Army 7- by 10 -Foot Wind Tunnel at NASA Ames Research Center.

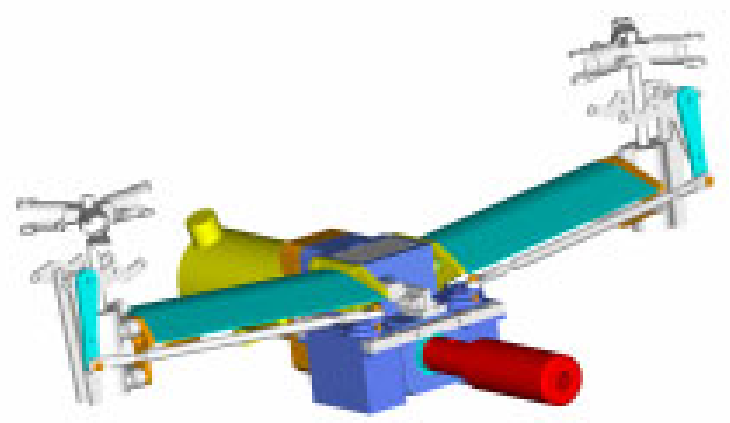

Figure 2. Drawing of tiltrotor model (wind tunnel flow from left to right).

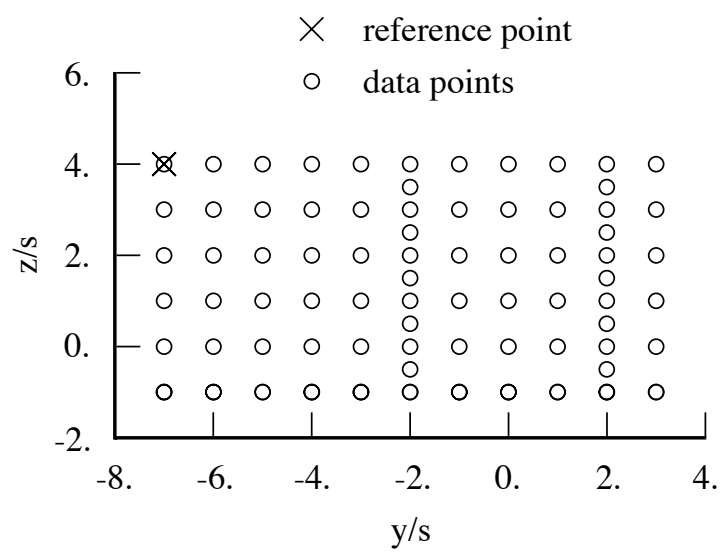

Figure 3. Grid of measurement locations (position of upwind aircraft relative to downwind aircraft). 


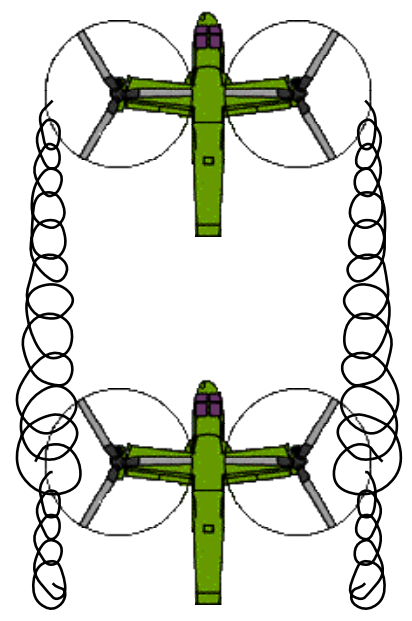

(a) $y / s=0$

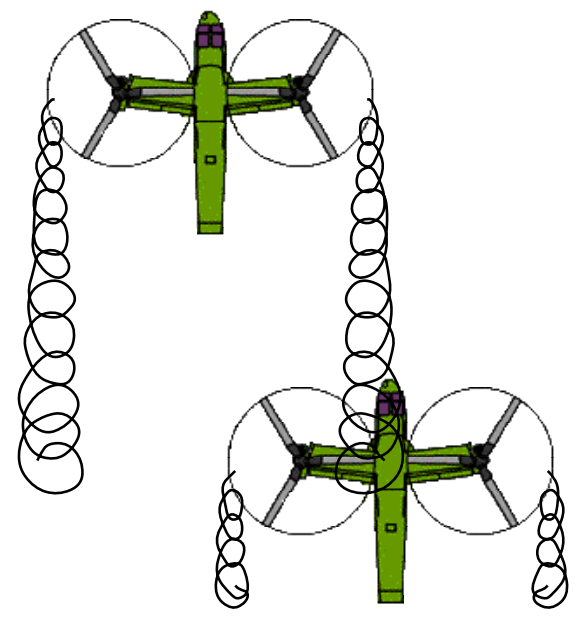

(b) $y / s=-2$

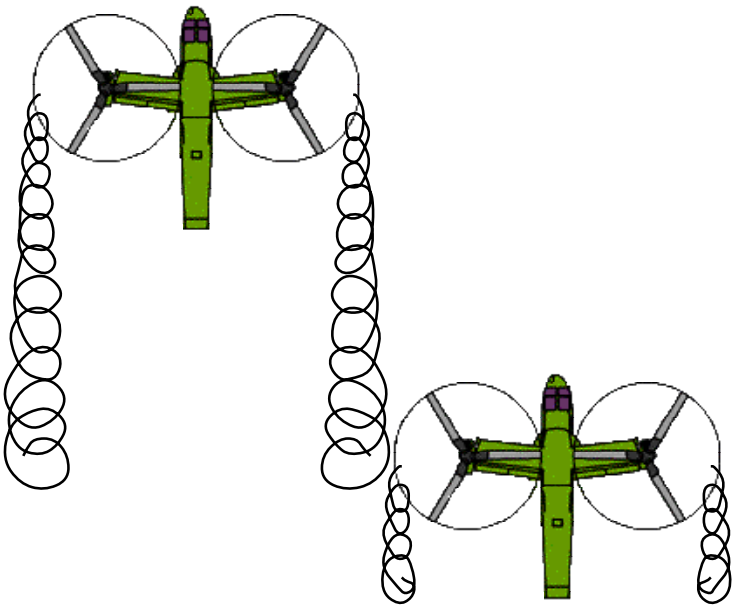

(c) $y / s=-4$

Figure 4. Illustrations of the relative positions of the two aircraft.

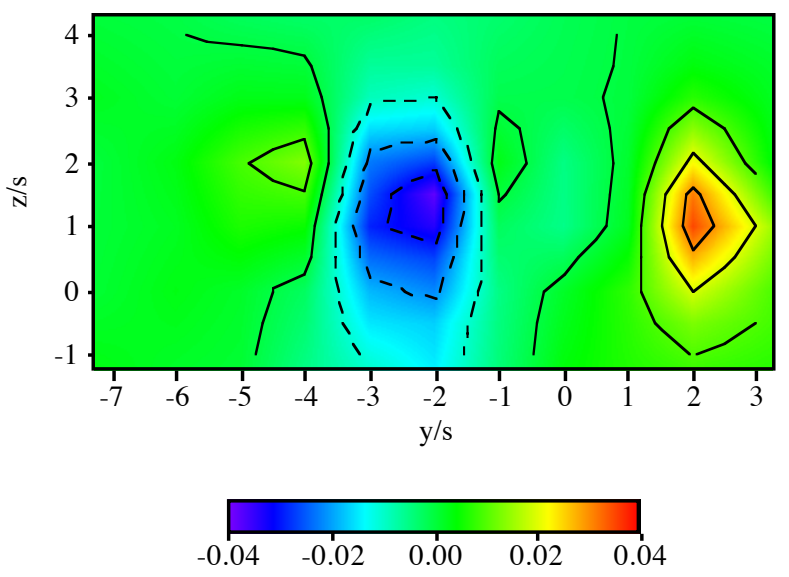

(a) $\mathrm{x} / \mathrm{D}=10.0$

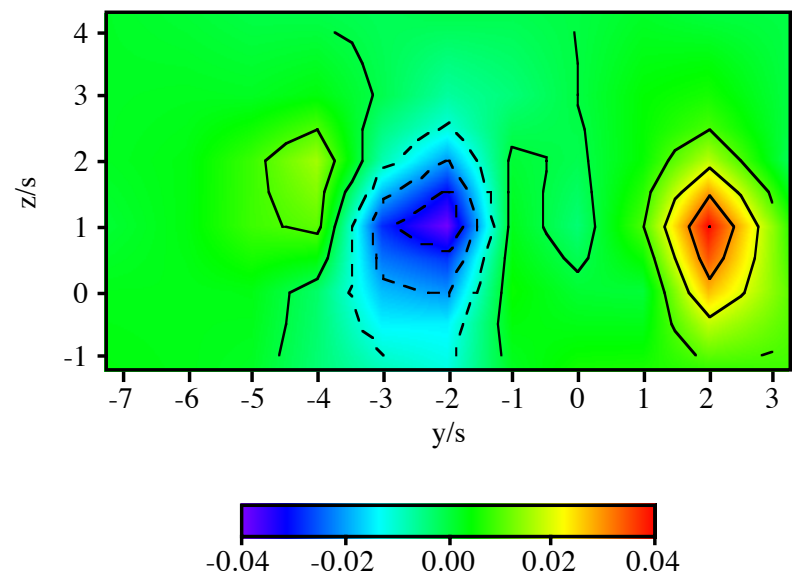

(b) $\mathrm{x} / \mathrm{D}=5.0$

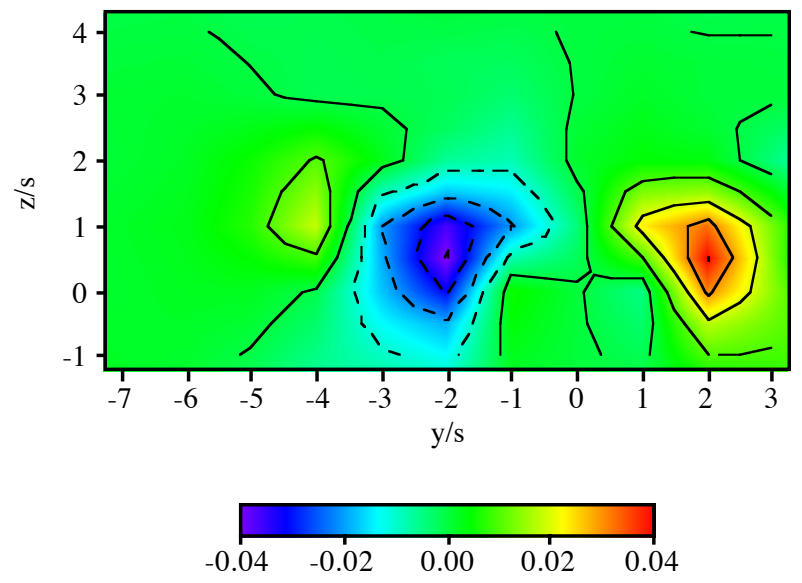

(c) $\mathrm{x} / \mathrm{D}=2.5$

Figure 5. Measured roll moment coefficient $\mathrm{C}_{\mathrm{Mx}} / \sigma$ of downwind aircraft, at $\mu=0.1$, as function of upwind aircraft position (contour increment $=0.01$, dashed line for negative). 

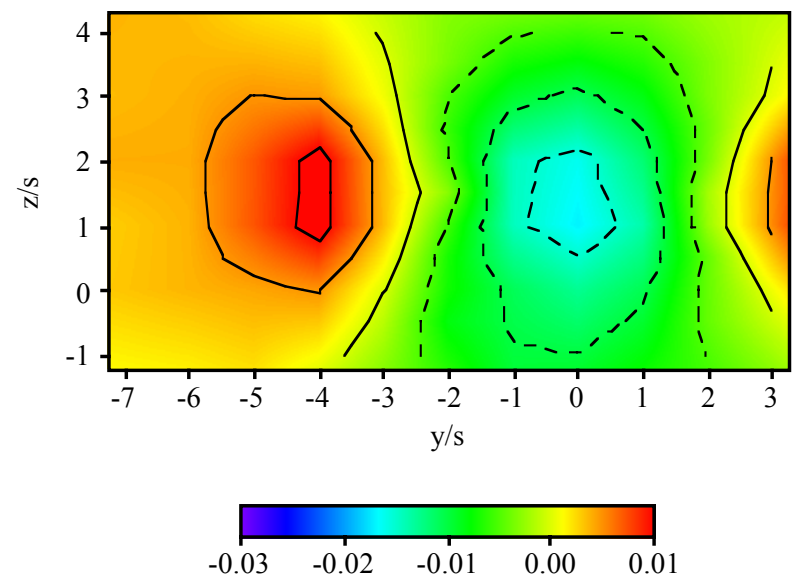

(a) $x / D=10.0$
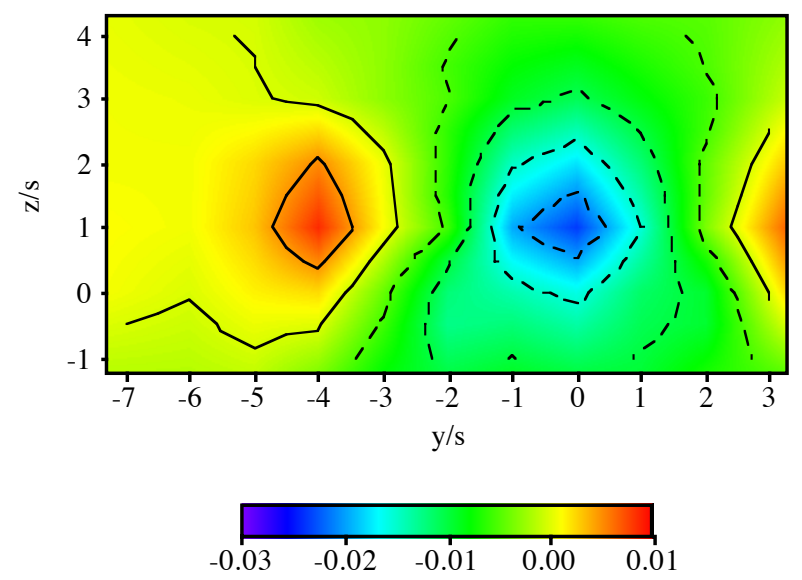

(b) $\mathrm{x} / \mathrm{D}=5.0$
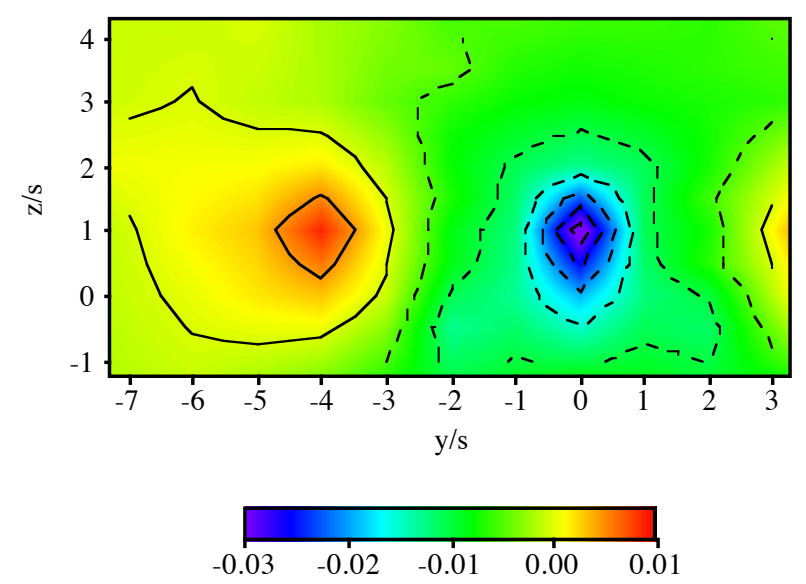

(c) $\mathrm{x} / \mathrm{D}=2.5$

Figure 6. Measured thrust change $\Delta \mathrm{C}_{\mathrm{T}} / \sigma$ of downwind aircraft, at $\mu=0.1$, as function of upwind aircraft position (contour increment $=0.005$, dashed line for negative).
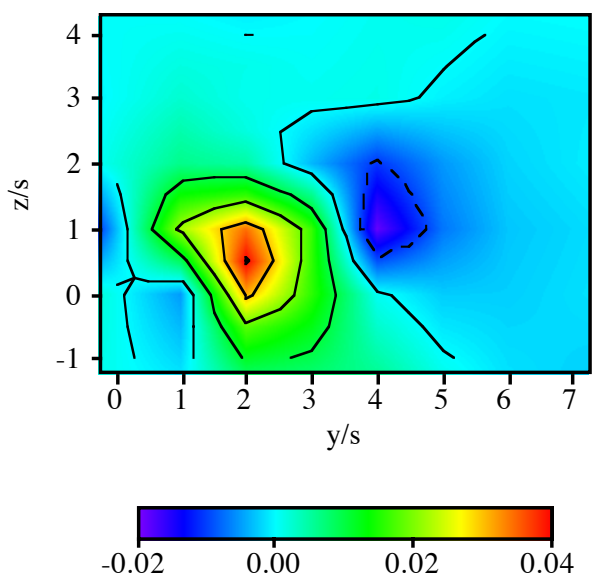

(a) measured

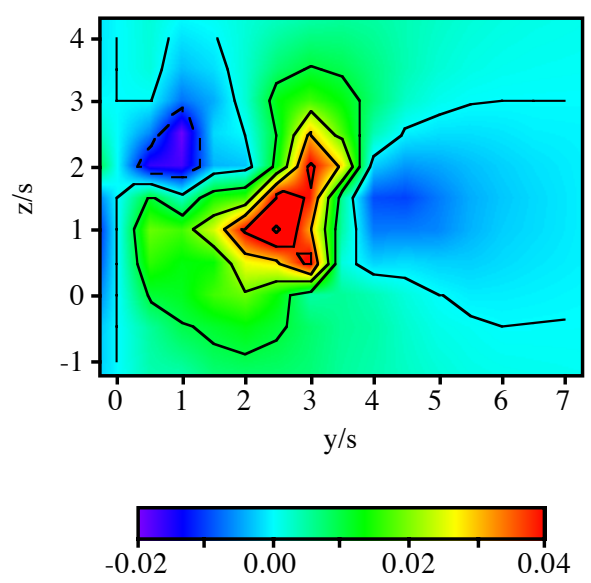

(b) calculated

Figure 7. Comparison of measured and calculated roll moment coefficient $\mathrm{C}_{\mathrm{Mx}} / \sigma$ of downwind aircraft, at $\mu=$ 0.1 and $\mathrm{x} / \mathrm{D}=2.5$ (contour increment $=0.01$, dashed line for negative). 

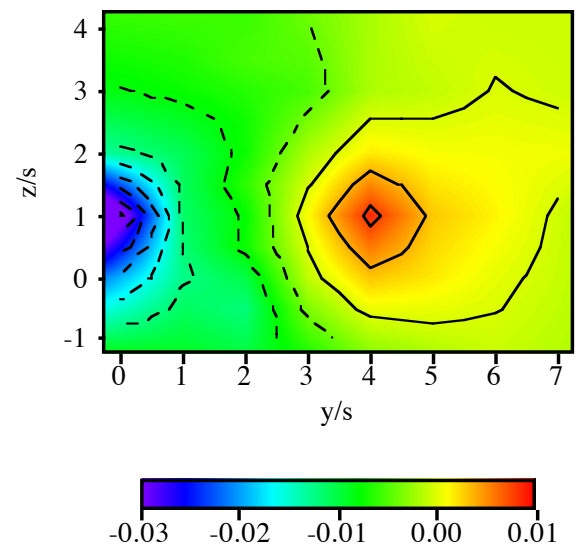

(a) measured

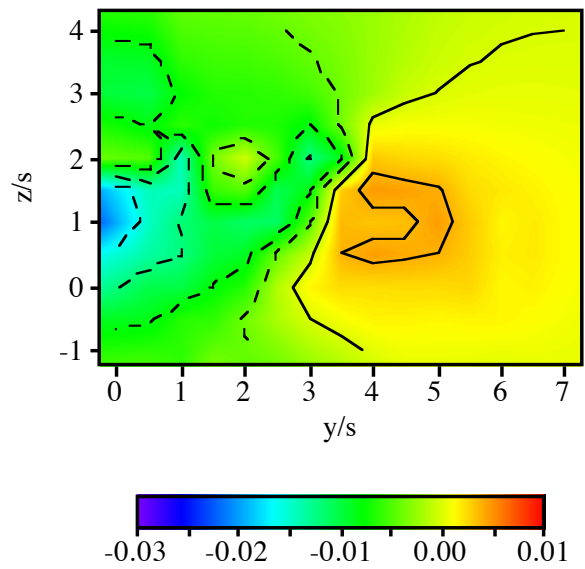

(b) calculated

Figure 8. Comparison of measured and calculated thrust change $\Delta \mathrm{C}_{\mathrm{T}} / \sigma$ of downwind aircraft, at $\mu=0.1$ and $\mathrm{x} / \mathrm{D}$ $=2.5$ (contour increment $=0.004$, dashed line for negative).

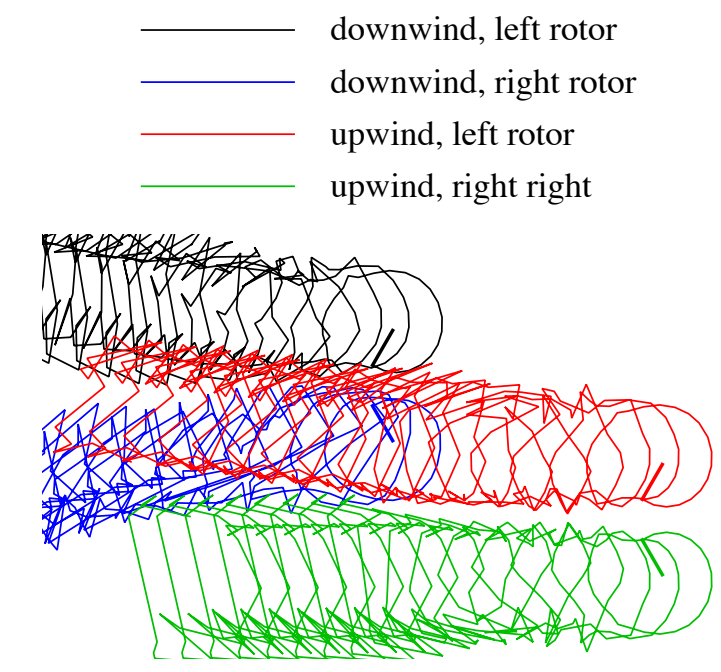

a) top view

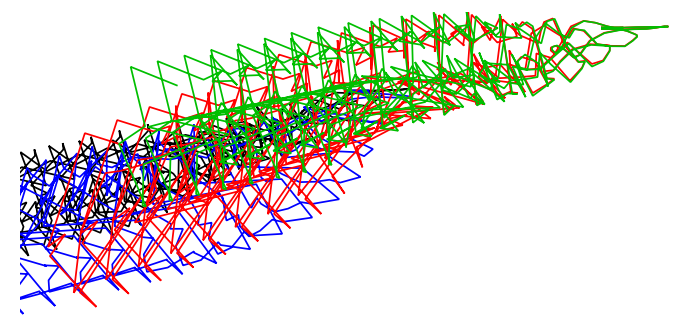

b) view from starboard

Figure 9. Calculated wake geometry, at $\mu=0.1, \mathrm{x} / \mathrm{D}=$ $2.5, \mathrm{y} / \mathrm{s}=2, \mathrm{z} / \mathrm{s}=1$ (only one tip vortex from each rotor shown). 


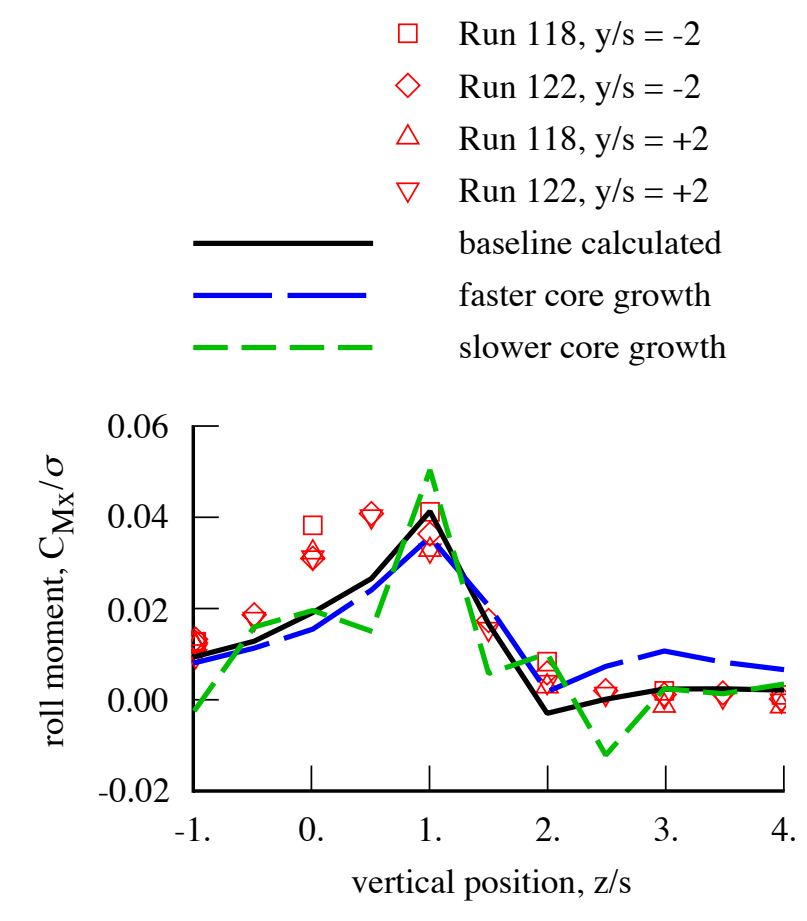

Figure 10. Influence of tip vortex core growth on calculated roll moment of downwind aircraft, at $\mu=0.1$, $\mathrm{x} / \mathrm{D}=2.5, \mathrm{y} / \mathrm{s}= \pm 2$.
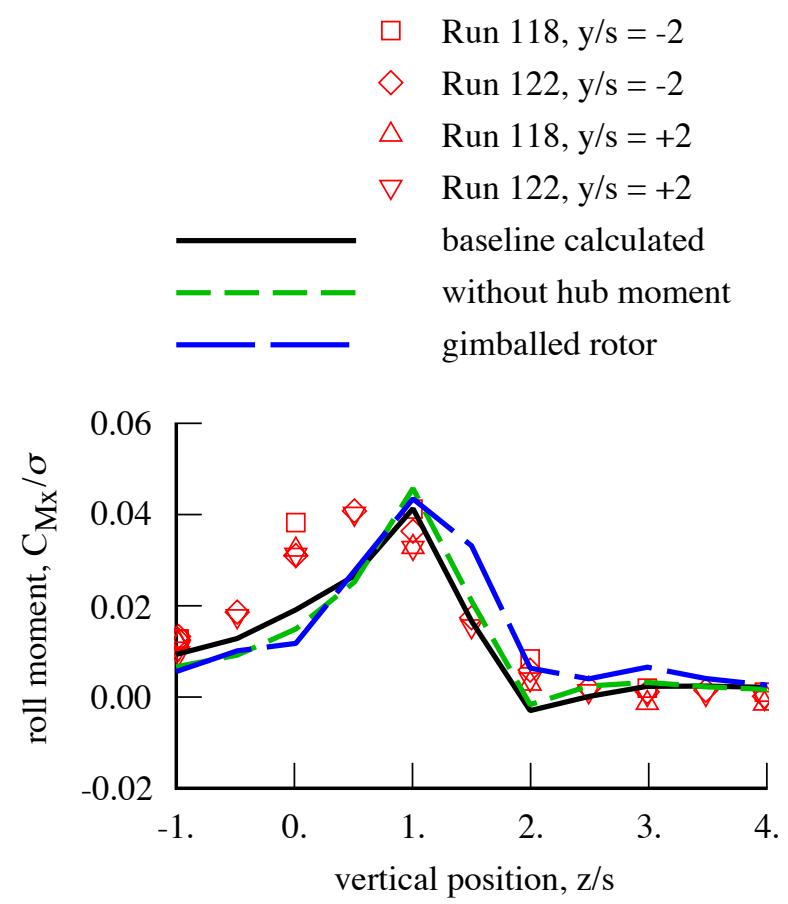

Figure 11. Influence of hub moment on calculated roll moment of downwind aircraft, at $\mu=0.1, \mathrm{x} / \mathrm{D}=2.5$, $\mathrm{y} / \mathrm{s}= \pm 2$.
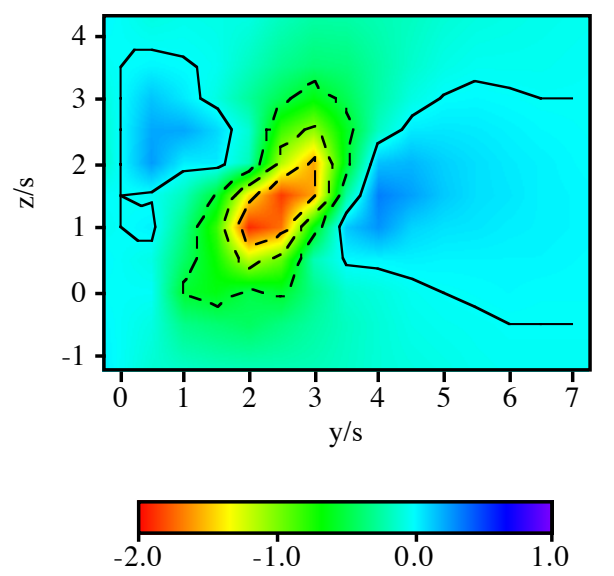

(a) lateral control (differential collective, deg)
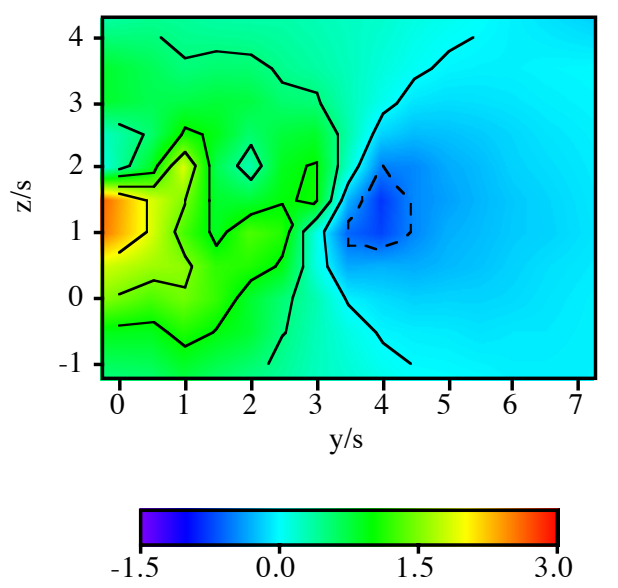

(b) collective change (deg)

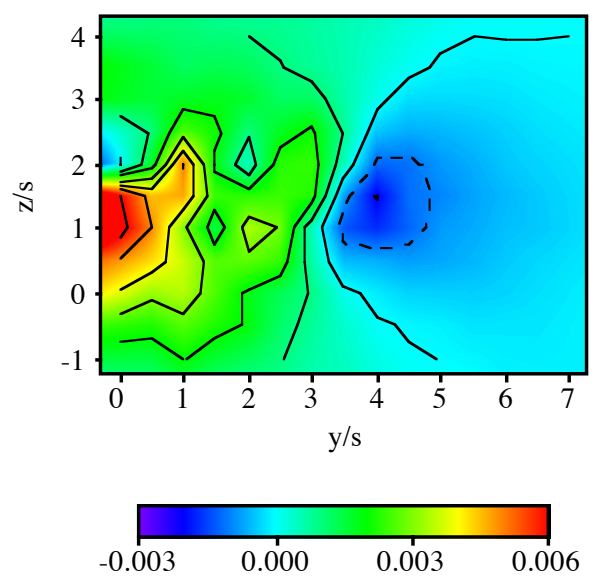

(c) power change, $\Delta \mathrm{CP}_{\mathrm{P}} / \sigma$

Figure 12. Calculated control and performance changes of downwind aircraft, when trimmed to specified thrust and zero roll moment, at $\mu=0.1$ and $\mathrm{x} / \mathrm{D}=2.5$ (contour increment $=0.5 \mathrm{deg}$ for control, 0.001 for power coefficient; dashed line for negative). 